\title{
Persistência e Mudança de Temas na Estruturação do Campo Científico da Estratégia em Organizações no Brasil(1)
}

\author{
Clóvis L. Machado-da-Silva \\ Luciano Rossoni
}

\section{RESUMO}

No presente artigo, procuramos verificar como o nível de coesão estrutural dos pesquisadores da área de estratégia no Brasil condiciona a construção do conhecimento científico nesse campo social. Por meio da análise de agrupamentos, levada a efeito mediante uso da técnica de análise de redes, foram avaliados 2.332 artigos publicados em periódicos e anais de congressos científicos brasileiros entre os anos de 1997 e 2005 . Com base na vertente estruturacionista da perspectiva institucional de análise examinamos o papel das relações entre autores na persistência e na mudança de temas no decorrer do tempo. Verificamos que o crescimento significativo do número de trabalhos no campo da estratégia foi condicionado em termos de temas pelos agrupamentos formados em momentos anteriores. Observamos alto grau de homogeneidade dentro dos agrupamentos mesmo em face da ocorrência de heterogeneidade de temas de pesquisa no campo como um todo. Tal constatação indica que a imersão em grupos sociais condiciona o que é legitimamente aceito no campo científico, implicando em sistemas de significado específicos em cada agrupamento, apesar da ocorrência de certo nível de comunalidade entre os integrantes do campo. Esse achado tem óbvias implicações para a conversação entre diferentes grupos de autores.

Palavras-chave: teoria institucional; estruturação; redes sociais; campo científico; estratégia em organizações.

\begin{abstract}
In this article we seek to verify how the level of structural cohesion of researchers in the field of strategy in Brazil conditions the construction of scientific knowledge in this social field. Through the analysis of clusters, taken to effect through the use of the network analysis technique, 2,332 articles were evaluated which were published in Brazilian periodicals and annals of scientific congress from 1997 to 2005 . Based on the structurationist perspective of the institutional theory, we examined the role of relations between authors in the persistence and changes in themes over time. We found that the significant growth in the number of papers in the field of strategy was conditioned in terms of themes by the clusters that had been previously formed. We observed a high degree of homogeneity within the clusters even with the occurrence of heterogeneity of themes of research in the field as a whole. This indicates that embeddedness in social groups conditions what is legitimately accepted in the scientific field, implying that there are specific systems of meaning in each cluster, despite the occurrence of a certain level of communality among members of the field. This finding has obvious implications for conversation between different groups of authors.
\end{abstract}

Key words: institutional theory; structuration; social networks; scientific field; strategy in organizations. 


\section{INTRODUÇÃO}

O campo científico, como qualquer campo social, é um sistema social como demonstram Machado-da-Silva, Guarido Filho e Rossoni (2006). Como tal apresenta "relações reproduzidas entre atores ou coletividades, organizadas como práticas sociais regulares" (Giddens, 1989, p. 20), o que possibilita que seu componente estrutural seja analisado como uma ampla rede social (Moody, 2004; Powell, White, Koput, \& Owen-Smith, 2005; Wagner \& Leydesdorff, 2005; White, Owen-Smith, Moody, \& Powell, 2004). No entanto, campos e redes sociais têm sido analisados, usualmente, de maneira isolada um do outro, apesar do potencial que há em sua junção para o entendimento de como campos institucionais se desenvolvem (De Nooy, 2003; Powell et al., 2005).

Nesse sentido, o objetivo do presente trabalho é compreender o campo científico da Estratégia em Organizações mediante a confluência das noções de campos e de redes sociais. Para tanto, verificamos a estrutura de relações entre autores em conexão com a produção científica deles, visando a entender as instituições sociais que conferem identidade ao campo de conhecimento em estratégia na área de administração no contexto brasileiro. Estudo semelhante foi desenvolvido por Déry e Toulouse (1996), que usaram análise de redes e co-citações para avaliar a estruturação do campo de empreendedorismo. Todavia, os autores pouco avançaram na interpretação de seus achados sob um ponto de vista estruturacionista.

O pressuposto no presente trabalho é que as relações entre autores estão conectadas com a produção do conhecimento científico no campo da estratégia, o que pode ser evidenciado pela análise das práticas de pesquisa vigentes, uma vez que se encontram objetivadas no conteúdo científico dos artigos. Nesse ponto emerge uma das mais importantes questões do estudo: como tratar das relações entre autores e do conteúdo da produção científica de tal forma que possam garantir e elucidar uma avaliação institucional do fenômeno em exame?

Nessa direção, adotamos um recorte longitudinal para aproximar o entendimento da dinâmica das redes de relacionamentos com a construção do conhecimento científico na área de estratégia. A partir dos dados coletados em 2.332 artigos publicados em periódicos e anais de eventos científicos brasileiros, classificados como sendo de nível "A" no sistema Qualis da CAPES, no período compreendido entre os anos de 1997 e 2005, identificamos 2.072 autores que compõem o campo de pesquisa em estudos organizacionais e estratégia no Brasil. Desse total, 
separamos 1.128 autores em um primeiro momento: 835 que publicaram exclusivamente na área de estratégia e 293 que publicaram em ambas as áreas. A partir daí destacamos um número menor com base tão somente nas relações que demonstraram ser relevantes na estruturação do campo de pesquisa em estratégia no país.

Vale destacar que mesmo apresentando predominantemente dados quantitativos no presente estudo, procuramos por meio da avaliaçao do conteúdo dos artigos produzidos entender as estruturas cognitivas dos autores enquadrados em um grupo com base na análise das suas relações com outros autores. Ainda que o contéudo dos artigos seja algo já objetivado, ele fornece elementos importantes sobre o entendimento do que seja ciência na visão dos autores. Além disso, permite que se verifique o que eles realmente praticam (fazem) como ciência. Ressaltamos, entre outras possíveis, uma limitação deste tipo de análise: autores solitários praticamente não são considerados ainda que apresentem produção científica numerosa.

Na seqüência, o presente artigo está estruturado em quatro partes. Após esta breve introdução, contendo o objetivo do estudo, apresentamos o quadro teórico de referência. Na terceira parte descrevemos os procedimentos metodológicos que sustentam a parte empírica do trabalho. A quarta parte abrange a apresentação e a análise dos dados. Por fim, apresentamos as conclusões que decorrem da interpretação dos dados à luz do quadro de referência conceitual utilizado.

\section{Quadro Teórico de Referência}

Desde o trabalho seminal de Granovetter (1985) sobre imersão social (embeddedness), uma série de estudos foram realizados para avaliar o papel das relações entre atores sociais na construção de significados, levando muitos institucionalistas a considerar os sistemas relacionais como condutores das instituições nas sociedades humanas (Scott, 2001). Na perspectiva da sociologia do conhecimento, Leydesdorff (2007) realça a importância do entendimento das relações entre autores de trabalhos científicos para se compreender a sua cognoscitividade, destacando a existência de padrões estruturados de cognição. Estruturas cognitivas condicionam e são condicionadas pelas relações entre atores sociais mediante o compartilhamento de significados, ou seja, pela intersubjetividade que se estabelece entre eles. Tal intersubjetividade pode ser tratada como uma objetividade espaciotemporalmente delimitada no entendimento de Machado-daSilva, Fonseca e Crubellate (2005). 
A partir dessa linha de raciocínio, cria-se a expectativa de que as relações entre autores de trabalhos científicos em determinada área de conhecimento vinculam-se com a própria produção do conhecimento naquele campo. Assim, mediante o uso da análise de redes pode-se verificar níveis mais abrangentes ligados à integração social e à integração de sistema dos autores em um campo científico, possibilitando descortinar caminhos para a identificação das estruturas cognitivas vigentes e dos limites da cognoscitividade.

A propósito, Giddens (1989) faz algumas observações genéricas sobre a tarefa da pesquisa social com base na teoria da estruturação. $\mathrm{O}$ autor afirma que a inserção metodológica do investigador pode ser feita em diferentes níveis, de acordo com o objetivo da pesquisa. Porém, para uma avaliação mais plena, tanto a consciência discursiva como a consciência prática devem ser melhor exploradas para se compreender a ordem institucional do campo científico.

Ademais, ele afirma que qualquer forma de significado levantado na análise sociológica pressupõe um momento hermenêutico pois serve para elucidar a cognoscitividade dos agentes envolvidos. Tal análise não precisa, necessariamente, apoiar-se em pesquisa de caráter qualitativo. Segundo ele, "a coleta e interpretação de material quantitativo depende de procedimentos metodológicamente idênticos à coleta de dados de uma espécie "qualitativa' mais intensiva" (Giddens, 1989, p. 270). Dessa forma, ele entende que desaparece a nítida distinção entre métodos qualitativos e quantitativos em pesquisas estruturacionistas, pois trabalhos contendo dados quantitativos sofrem interpretações qualitativas. O que efetivamente importa, nessa perspectiva, é a interligação entre as bases ontológicas e epistemológicas da explicação teórica, em face do papel que se atribui ao pensamento na construção do conhecimento científico.

Além dessa questão, é amplamente disseminada no mundo acadêmico a crença de que grandes inovações no campo científico decorrem de rupturas radicais. Tal visão é atribuída principalmente ao trabalho de Kuhn (1978) nas ciências exatas e biomédicas. A transposição das idéias de Kuhn para as ciências sociais é vista com certa cautela por muitos estudiosos, a ponto de Giddens (1978) afirmar que os paradigmas eram vistos por Kuhn como sistemas fechados. Na lógica da teoria da estruturação, as inovações em um campo científico referemse à (re)produção de práticas vigentes, uma vez que são interpretadas e transformadas por agentes, gerando novas práticas institucionalizadas. Assim, sempre haverá um movimento recursivo entre estruturas institucionais e ações, configurando a noção de dualidade da estrutura, que é amplamente tratada por Giddens (1989).

Nessa perspectiva, reconhecemos que qualquer processo de mudança no campo científico sempre acontece a partir das estruturas institucionais vigentes, sendo 
elas (re)produzidas especialmente por meio da prática de pesquisa objetivada sob a forma de artigos científicos. No entanto, como a abordagem da realidade nas ciências sociais não se restringe a uma única forma, mas, muito pelo contrário, há diferentes maneiras de abordá-la, como é possível entender o desenvolvimento de tais perspectivas diante do crescimento acelerado por que vem passando as diferentes áreas científicas, nos últimos anos?

Na literatura especializada sobre redes sociais, coloca-se que mecanismos ligados à estrutura de relacionamentos interferem no processo de institucionalização das ações sociais. Em face dessa menção, acreditamos que a produção científica tenha uma relação dual com a configuração reticular do campo. Como afirma Giddens (1989), as propriedades estruturais dos sistemas sociais (aqui tratados como campos científicos) constituem tanto meio como fim das ações dos agentes: meio quando servem de suporte para a realização da ação e fim quando as propriedades são (re)produzidas a partir da prática (Machado-da-Silva et al., 2006).

De acordo com DiMaggio e Powell (1983) e Scott (1994, 2001), campos organizacionais (campo científico neste artigo) subentendem uma área reconhecida de vida institucional em que os atores sociais (autores neste estudo) compartilham de sistemas de significados comuns, possibilitando o isomorfismo entre os participantes do campo. Esses padrões isomórficos necessários à estruturação de um campo contribuem para certa homogeneização de práticas. Tais práticas são compartilhadas e reproduzidas a partir dos programas de pós-graduação, dos encontros acadêmicos formais e informais, e dos sistemas de avaliação instaurados na pós-graduação, seja de avaliação da qualidade dos programas, seja na avaliação dos artigos submetidos para periódicos e anais de eventos científicos (Liberman $\&$ Wolf, 1997). Nesse sentido, entende-se que, mesmo havendo grande número de autores e grupos isolados, eles compartilham de certas crenças comuns, o que possibilita certo nível de conversação no campo, levando os autores à monitoração reflexiva da ação no sistema social.

Com suporte nos argumentos de Machado-da-Silva et al. (2005) de que a teoria institucional deve ser tratada como teoria multiparadigmática, em que os autores propõem a análise das redes de relações sociais com base em perspectiva longitudinal, procuramos no presente artigo entender como o nível de coesão estrutural dos autores interfere na construção do conhecimento científico no campo da estratégia. Tratamos como desafio o problema de conciliar um método fundamentalmente estruturalista (Freeman, 1992) com uma perspectiva estruturacionista da teoria institucional, que considera a construção social como fenômeno intersubjetivo estreitamente ligado com estruturas cognitivas no processo de interpretação e de compartilhamento de significados. 
Em face de tal desafio, as idéias expostas por Giddens (1989) na teoria da estruturação fornecem o arcabouço conceitual que possibilita o estudo da relação entre estrutura e agência de maneira dual, com ênfase nas noções de recorrência e de simultaneidade. A primeira dessas idéias consiste na possibilidade de visualizar as redes de relações entre os autores como constituídas pelas ações dos sujeitos (Giddens, 1978). Assim, as relações sociais não são vistas como dadas, mas como fruto da produção e da reprodução dos sistemas sociais por parte de agentes capacitados no campo. Tendo em vista que as relações sociais integram os sistemas sociais, elas são primordiais para a compreensão das ordens institucionais e dos limites da cognoscitividade humana (Giddens, 1989).

Nessa linha de raciocínio, mesmo analisando as relações entre os autores como algo já objetivado por meio das relações de cooperação na produção de artigos científicos, elas possibilitam a reconstrução de parâmetros de ação, que permitem, juntamente com a avaliação do conteúdo de tais relações, entender como os agentes (re)produzem as práticas de pesquisa no decorrer do tempo. Para isso, algumas distinções devem ser feitas. A primeira é relativa ao que se entende aqui como estrutura social. Em coerência com o arcabouço utilizado no presente estudo, vislumbra-se a estrutura social como virtual, existente somente como traços de memória (Giddens, 1989). Já por estrutura de relações entende-se o conjunto de relações diretas e indiretas desenvolvidas espaciotemporalmente, portanto delimitadas, que fazem parte do sistema social, aqui visto como campo de conhecimento de estratégia em organizações. Essas relações possibilitam a (re)produção das estruturas sociais, uma vez que limitam e habilitam as ações dos agentes. Em decorrência, ao circunscrever os autores do campo de estudo em estratégia mediante análise de suas relações esperamos entender como tais relações estão correlacionadas com a produção científica desses pesquisadores.

Ao se considerar o compartilhamento de crenças e valores no campo da estratégia e a heterogeneidade de perspectivas ontológicas, epistemológicas, teóricas e metodológicas, as relações de cooperação indicam que os autores adotam algum critério para escolha dos pares, selecionando autores com abordagens de seu interesse e que também lhe sejam acessíveis, ao tempo que detenham algum grau de legitimidade no campo como um todo. Segundo Moody (2004), há pouco consenso teórico nas ciências sociais, mas isso não sugere que, dentro de suas especialidades, não existam grupos de disciplinas.

Tendo em vista que as crenças e os valores do que é legitimamente aceito como conhecimento científico são construídas a partir das relações sociais que condicionam a visão de ciência dos autores (Fuchs, 1993; Leydesdorff, 2007), procuramos, na avaliação das relações entre eles e de suas respectivas produções científicas, entender como eles estão imbricados. Assim, o estudo partiu do pressuposto de que a dinâmica de relacionamento entre pesquisadores tanto 
influencia como é influenciada pelas práticas institucionalizadas de pesquisa. Portanto a dualidade entre estrutura de relações e prática de pesquisa reflete-se na construção do conhecimento científico. Como afirma Moody (2004), cientistas imersos em redes de colaboração compartilham ideias, usam métodos e técnicas similares, e influenciam o trabalho dos outros. Modelos de influência sugerem que cientistas trocam experiências, questões de pesquisa, métodos e regras explícitas e implícitas de avaliação com seus colaboradores. Portanto, as redes de coesão estrutural podem propiciar algum consenso, pelo menos no que diz respeito aos problemas e procedimentos utilizados (Friedkin, 1998).

Entender os grupos é importante, uma vez que eles podem fornecer a coesão necessária para a construção social do significado (Friedkin, 1998). No caso da produção científica, a construção de parâmetros de trabalho do que é ou não aceito como conhecimento é definido, em primeira instância, dentro dos grupos de pesquisadores. Compreendê-los possibilita entender como o isomorfismo opera na construção do conhecimento. Nesses grupos, existem autores que exercem maior influência do que outros. Ao identificar tais autores, é bem provável que se compreenda boa parte das questões de pesquisa de um grupo de pesquisadores.

Por conseguinte, sob a luz das afirmações de Kuhn (1978) e de Popper (1972, 1989) de que o conhecimento científico é construído socialmente, pressupomos no presente estudo que a configuração das relações no campo interfere na construção do conhecimento científico. No âmbito específico do objetivo deste trabalho, esperamos que as preferências sobre os temas dos artigos sejam orientadas pela construção de relacionamentos no decorrer do tempo, o que possibilita entender as implicações institucionais de tais relações.

Além do entendimento das configurações dos relacionamentos entre os autores procuramos compreender como ela se desenvolveu no decorrer do tempo. Pressupomos que a estrutura de relacionamento e a produção científica influenciam a construção de padrões de interação, longitudinalmente. Sob a perspectiva de que os relacionamentos são dinâmicos, buscamos compreendêlos a partir de reconstrução histórica, na qual a simultaneidade e a recursividade entre os elementos estão presentes no campo científico (Machado-da-Silva et al., 2005; Machado-da-Silva et al., 2006).

\section{Procedimentos Metodológicos}

O presente estudo é predominantemente de natureza descritiva, com utilização de método misto de pesquisa. Procuramos verificar os elementos estruturais da 
rede de relacionamento entre pesquisadores, mediante o uso da metodologia de análise de redes. Além disso, adotamos outros mecanismos de tratamento dos dados, como a análise documental e a análise de conteúdo, para verificar apropriadamente o conteúdo dos artigos publicados. O delineamento da pesquisa é o de análise de redes com uso de fontes de pesquisa documental: periódicos e anais de congressos científicos. Os dados coletados permitiram análises descritivas dos relacionamentos entre autores com alguns desdobramentos explicativos concernentes a certos aspectos. A perspectiva temporal de análise é longitudinal, o que possibilitou o exame da dinâmica de relacionamento entre os autores e a verificação de mudanças estruturais no campo em termos de coesão de grupos no decorrer do tempo (Moody, 2004; Powell et al., 2005; Wasserman \& Faust, 1994; White et al., 2004). O nível de análise da pesquisa é a rede de autores da área de estratégia em organizações e as unidades de análise são os agrupamentos de pesquisadores com a correspondente identificação dos autores líderes, quando claramente evidente. Os procedimentos de pesquisa empregados foram tanto quantitativos como qualitativos. Em termos quantitativos, utilizamos especificamente o aparato estatístico da análise de redes. As análises documentais e de conteúdo foram tratadas tanto de forma quantitativa como qualitativa, com preponderância para esta última.

Amostragem, Coleta e Tabulação dos Dados. Tendo em vista o foco de interesse na coesão estrutural do campo da estratégia, a partir da rede de relações entre autores, escolhemos como unidade amostral cada artigo publicado na área de Estratégia em Organizações em anais de eventos e em periódicos classificados como nível ‘A Nacional' pelo Sistema Qualis da Capes, no período compreendido entre 1997 e 2005. Três eventos científicos foram considerados, todos eles vinculados à ANPAD - Associação Nacional de Pós-Graduação e Pesquisa em Administração: Encontro da ANPAD (EnANPAD), Encontro de Estudos Organizacionais (EnEO) e Encontro de Estudos em Estratégia (3Es). Os sete periódicos consultados foram os seguintes: Revista de Administração Contemporânea (RAC), Revista de Administração de Empresas (RAE), Revista de Administração de Empresas Eletrônica (RAE-Eletrônica), Revista de Administração da Universidade de São Paulo (RAUSP), Revista Organizações \& Sociedade (O\&S), Revista de Administração Pública (RAP) e Revista Eletrônica de Administração (REAd).

A partir das unidades amostrais chegamos a cada autor que, sozinho ou em conjunto com outros autores, publicou algum artigo científico. Essa verificação consistiu basicamente em identificar todas as relações de co-autoria que um autor manteve no período estudado. Os dados coletados, tanto os relacionais como os atributos, foram tabulados com a ajuda dos softwares Microsoft Excel ${ }^{\circledR}$, UCINET 6.0 (Borgatti, Everett, \& Freeman, 2002) e PAJEK 1.10 (Batagelj \& Mrvar, 
2005). Estes softwares também foram utilizados como importantes auxiliares na análise dos dados conforme explicamos na seqüência.

Procedimentos para Análise dos Dados. A análise dos dados foi quantitativa, relacional, categórica e qualitativa. Para avaliar o processo de construção social do conhecimento científico procuramos relacionar a estrutura da rede com o conteúdo construído pelos autores na área de estratégia, longitudinalmente, levando em conta três subconjuntos temporais: 1997-1999; 2000-2002; e 2003-2005. Para tanto, selecionamos os grupos por meio da medida $n$-clan, em que o diâmetro máximo do subgrafo é exclusivamente menor ou igual a $n$ (Hanneman $\&$ Riddle, 2005; Wasserman \& Faust, 1994), o que possibilita que os grupos formados possam ser considerados realmente coesos, uma vez que esta medida classifica os autores por estarem em uma mesma esfera de influência. Segundo Hanneman e Riddle (2005), a medida $n$-clan freqüentemente é bem ajustada para dados sociológicos, o que reforçou a nossa opção por ela. Escolhemos para observação grupos com sete ou mais autores e com diâmetro máximo de dois.

Com os grupos identificados, adotamos um método de agrupamento de acordo com a similaridade para evitar redundância nas análises. Muitos artigos apresentam até seis autores, o que por si só forma cliques de até seis componentes com um único artigo. Como o objetivo da análise de coesão de grupos, no presente estudo, é verificar graus de persistência na colaboração entre autores, decidimos examinar os grupos a partir de sete autores. Desse modo, diminuímos o efeito de artigos com grande número de co-autores.

A identificação dos artigos produzidos pelos autores de cada grupo no período, em termos de áreas, eixos temáticos e temas principais foi levada a cabo mediante uso de técnicas de análise de conteúdo. O critério de categorização utilizado foi o semântico (categorias temáticas), em que os temas com mesmo significado são agrupados na mesma categoria. Depois do inventário, adotamos o procedimento de categorização por 'milha', que é feita a posteriori, isto é, resulta da análise do conteúdo científico dos artigos. Realizamos todo o processo de análise predominantemente de forma qualitativa, com uso do software Microsoft Excel ${ }^{\circledR}$, pois verificamos somente a presença e a ausência de temas a partir dos títulos, resumos e conteúdo dos artigos publicados, mesmo sendo a frequiência também levada em consideração. Com esse procedimento foi possível identificar o grau de homogeneidade e de heterogeneidade da produção científica, mediante a comparação com a formação estrutural dos grupos.

As análises foram realizadas com base nos três triênios já mencionados: 19971999; 2000-2002; e 2003-2005. A escolha da divisão trienal foi logicamente factível: possibilitar a comparação em termos reais a partir de frações do período total 
compostas pelo mesmo número de anos. Além disso, permitiu evitar possíveis efeitos sazonais dos eventos na área de estratégia, facilitando a interpretação dos resultados.

Com o objetivo de orientar a análise da produção científica da área de estratégia, ela foi tratada em termos de subáreas. Para tanto, adotamos a classificação utilizada no EnANPAD de 2005. No evento daquele ano, a Divisão de Estratégia em Organizações, que havia sido instituída no ano imediatamente anterior, considerou três áreas temáticas: Estratégia em Organizações (ESO), Gestão Internacional (GIN), e Empreendedorismo e Comportamento Empreendedor (EMP). Há, sem dúvida, outras classificações em termos de subáreas da área de Estratégia em Organizações: algumas melhores; outras piores. Preferimos fazer uso daquela que orienta a submissão de artigos científicos na academia brasileira de administração.

\section{A Situação em Estudo: Análise dos Resultados}

Os diagramas de árvore, que indicam o agrupamento dos autores, podem ser visualizados na Figura 1. Eles permitem esclarecer quais são os grupos que compõem a estrutura de relacionamento no campo de pesquisa em Estudos Organizacionais e Estratégia em Organizações, nos três triênios em exame. Apesar de a coleta de dados haver englobado essas duas áreas de conhecimento, circunscrever-nos-emos ao campo da Estratégia em Organizações na análise da persistência ou mudança de temas no período de 1997 a 2005.

Figura 1: Agrupamentos Formados por meio da Medida $\mathrm{N}$-clan

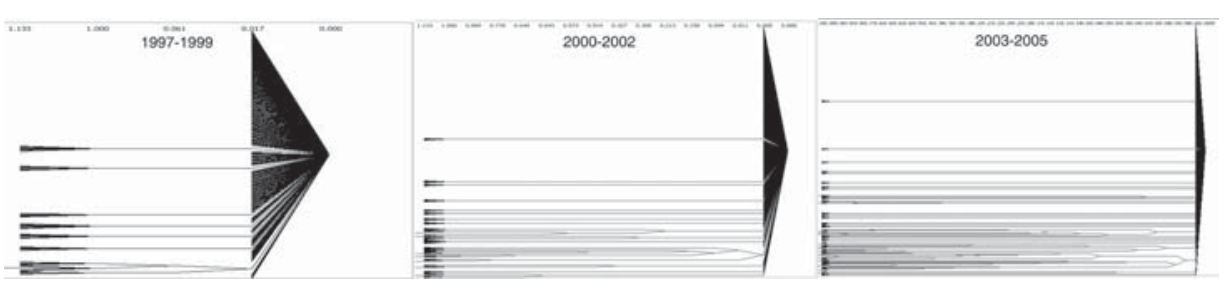

No primeiro triênio, observamos somente nove $n$-clans que apresentam sete ou mais autores; no segundo, encontramos 28; e, no terceiro, 66. Tais dados demonstram a tendência de aumento no número de agrupamentos entre autores no campo da estratégia em organizações, no período total em estudo. Ao compararmos o crescimento no número de grupos (n-clans) com o número de autores por triênio, verificamos que o índice de crescimento no número de grupos 
foi bastante superior. Tais indicadores provavelmente foram influenciados pela tendência de maior colaboração entre autores, acarretando, crescentemente, maior número de grupos.

A rede contou com 387 autores no triênio 1997-1999, mas a grande maioria não estava ligada a qualquer tipo de grupo informal ou formal de pesquisadores. Contabilizamos 72 participantes nesses grupos, aproximadamente $19 \%$ do total de autores. Identificamos a média de 8,11 autores por grupo. Não custa registrar que não foram computados aqueles grupos com menos de sete autores. Os maiores grupos apresentaram nove autores e encontramos apenas três grupos com esse número.

Vale lembrar que autores podem participar de mais de um grupo. Como o objetivo do presente estudo é verificar a possível conexão entre rede de relações e temas de produção científica, não faz sentido considerar autores que estão isolados ou que colaboram com um número reduzido de co-autores. Apesar da identificação de nove grupos nesse triênio, três deles destacaram-se pela similaridade, com diversos autores participando de mais de um deles. Podemos visualizar no Quadro 1 os agrupamentos (clusters) formados por pesquisadores da área de estratégia em organizações nos anos de 1997 a 1999.

\section{Quadro 1: Agrupamentos e Principais Temas no Triênio 1997-1999}

\begin{tabular}{|l|l|l|l|}
\hline $\begin{array}{l}\text { Principais } \\
\text { instituições }\end{array}$ & Áreas & $\begin{array}{l}\text { Eixo temático } \\
\text { predominante }\end{array}$ & Temas mais abordados \\
\hline PUC-Rio & ESO & ESO & Estratégia e Vantagem Competitiva; Tipologias Estratégicas. \\
\hline UFBA & ESO & ESO & Administração Estratégica e Reestruturação Produtiva. \\
\hline UFPR & $\begin{array}{l}\text { ORG e } \\
\text { TEO }\end{array}$ & TEO e ESO & $\begin{array}{l}\text { Teoria Institucional e Estratégia; Estratégia e Competitividade; } \\
\text { Cognição e Estratégia; Estratégia e Mudança. }\end{array}$ \\
\hline
\end{tabular}

Fonte: Dados primários da pesquisa.

O primeiro agrupamento listado no Quadro 1 foi liderado pelo Professor Jorge Ferreira da Silva, da Pontifícia Universidade Católica do Rio de Janeiro (PUCRio), que trabalhou o conceito de estratégia sob perspectiva mais porteriana, privilegiando variáveis quantitativas em estudos sobre vantagem competitiva e, também, no desenvolvimento de tipologias estratégicas. O segundo grupo de estudos com foco em estratégia no triênio desenvolveu-se na Universidade Federal da Bahia (UFBA), tendo o Professor Rogério Quintella como condutor das pesquisas. Diferentemente do primeiro, este agrupamento analisou o fenômeno sob perspectiva mais qualitativa, mas ainda mantendo o pressuposto de racionalidade da escola clássica. $\mathrm{O}$ terceiro agrupamento, sob a liderança do Professor Clóvis L. Machado-da-Silva, da Universidade Federal do Paraná (UFPR), procurou conectar, em grande medida, as áreas de Organizações e Estratégia, ao tratar de temas que estão na confluência dos dois campos de 
conhecimento, como se pode observar no Quadro 1. Tanto procedimentos quantitativos como qualitativos de pesquisa são privilegiados por esse grupo.

Em linhas gerais, verificamos grande homogeneidade de temas dentro de cada agrupamento, o que reforça a afirmativa de que a coesão dos autores influencia o conteúdo por eles desenvolvido (Hanneman \& Riddle, 2005; Moody, 2004), constituindo um facilitador de práticas isomórficas de pesquisa. $\mathrm{O}$ isomorfismo delimita a construção de regras e de normas no campo (Scott, 2001), muitas delas vinculadas não só aos grupos, mas também a algumas organizações em especial, tais como universidades.

Observamos, ainda, que a organização de vínculo de trabalho dos autores contribui no sentido deles procurarem perspectivas semelhantes para abordarem a realidade. No entanto, a proximidade espacial não é condição sine qua non, uma vez que o eixo temático e os temas de escolha do autor influenciam fortemente a escolha de colaboradores. Por outro lado, ao direcionarmos o nosso foco no sentido de visualizar comparativamente os diversos grupos, já é possível observar alto grau de heterogeneidade de temas no campo, ainda quando apresentam pequeno número de autores. Todavia, a grande heterogeneidade de temas no campo não se faz acompanhar de correspondente conversação entre as abordagens, uma vez que os autores tendem a trabalhar nas perspectivas desenvolvidas somente no interior dos próprios grupos. Tal constatação demonstra que os grupos estão frouxamente conectados.

No segundo triênio, verificamos substancial incremento no número de grupos a partir da medida $n$-clan. Como a rede mais que dobrou de tamanho no triênio era de se esperar que mais grupos fossem formados. No entanto, o aumento de grupos com mais de sete autores foi proporcionalmente bem superior à ampliação no quantitativo de autores que passaram a integrar a rede. Observamos 28 grupos no triênio compreendido entre 2000 e 2002, com a média de 8,39 autores por grupo.

O aumento do número de laços médios por autor significa maior repercussão desses autores em mais grupos. Observamos que a média de autores por grupo também cresceu, além de haverem surgido grupos de maior tamanho. Diferentemente do triênio anterior, há grupos com tamanho relativamente superior ao número mínimo de integrantes estipulado: grupos que contam com 11, 12 ou 13 autores demonstram tendência de incremento no nível de cooperação entre eles para a produção de conhecimento científico.

Esses 28 grupos foram integrados por 235 autores, que corresponde a $30 \%$ da rede, demonstrando que, proporcionalmente, mais autores procuraram a colaboração entre si a partir da formação de grupos mais estruturados. Tal como no triênio anterior, vários desses grupos são muito similares. Em face disso, 
novamente utilizamos a análise de agrupamentos para selecionar um número menor de clusters para avaliação. Assim, identificamos 17 agrupamentos, dos quais oito são da área de estratégia; mais do que o dobro, portanto, do quantitativo verificado no triênio anterior.

No Quadro 2 é possível visualizar os oito agrupamentos encontrados no triênio 2000-2002. Esses clusters foram analisados com base nos mesmos procedimentos adotados para avaliação no triênio anterior. Algumas mudanças substantivas ocorreram nos agrupamentos identificados no segundo triênio. A primeira delas é o aparecimento de agrupamentos formados por autores de Instituições de Ensino Superior (IES) que não estavam presentes no campo no triênio anterior ou, ainda, que estavam trabalhando em grupos pequenos ou de forma solitária. Também observamos um número maior de instituições representadas por autores nos agrupamentos, evidenciando a ampliação das parcerias de cooperação interinstitucionais. Verificamos, ainda, aumento no número de agrupamentos que contam com mais de um grupo. No triênio anterior, somente um agrupamento apresentou mais de um grupo, contra seis deste segundo período.

\section{Quadro 2: Agrupamentos e Principais Temas no Triênio 2000-2002}

\begin{tabular}{|l|l|l|l|}
\hline $\begin{array}{l}\text { Principais } \\
\text { instituições }\end{array}$ & Áreas & $\begin{array}{l}\text { Eixo temático } \\
\text { predominante }\end{array}$ & Temas mais abordados \\
\hline UERJ & ESO & GIN & Controle Gerencial e Internacionalização. \\
\hline UFPR & $\begin{array}{l}\text { ORG } \\
\text { e ESO }\end{array}$ & TEO e ESO & $\begin{array}{l}\text { Teoria Institucional e Estratégia; Estratégia e Competitividade; } \\
\text { Cognição e Estratégia; Estratégia e Mudança. }\end{array}$ \\
\hline PUC-PR & ESO & ESO & Posicionamento Estratégico e Tecnologia. \\
\hline PUC-Rio & ESO & ESO & Tipologias Estratégicas e Estratégia Competitiva. \\
\hline $\begin{array}{l}\text { UFPR } \\
\text { FGV-RJ }\end{array}$ & ESO & ESO & Estratégia e Ambiente. \\
\hline UFPR & ESO & ESO & Conteúdo e Processo Estratégico. \\
\hline UFSC & ESO & ESO & Adaptação Estratégica e Capacidade Organizacional. \\
\hline FGV-SP & $\begin{array}{l}\text { ORG } \\
\text { e ESO }\end{array}$ & TEO e ESO & $\begin{array}{l}\text { Gestão de Organizações; Modismos Organizacionais; Simbolismo e } \\
\text { Pós-modernismo. }\end{array}$ \\
\hline
\end{tabular}

Fonte: Dados primários da pesquisa.

Além de constatarmos incremento no que concerne à diversidade de temas nos agrupamentos, uma mudança no período 2000-2002, em especial, merece destaque: o aumento do número de grupos que pesquisam nas áreas de estratégia e de organizações, simultaneamente. Ao que parece há crescente interesse dos autores em focar ambas as áreas, aproximando pesquisadores de diferentes bases conceituais.

Em comparação com o triênio 1997-1999, em que três agrupamentos dedicavamse à área de estratégia, neste segundo triênio encontramos oito agrupamentos. Dos oito agrupamentos interessados na área, verificamos que seis deles 
enquadram-se exclusivamente no eixo temático estratégia em organizações, com um deles, ligado à Universidade Estadual do Rio de Janeiro (UERJ), endereçando predominantemente o tema da internacionalização, porém com produção pequena, relativamente aos outros agrupamentos. Os outros dois clusters trabalharam na conexão entre as áreas de organizações e estratégia, ao tratar de temas que estão na confluência dos dois campos de conhecimento, utilizando tanto procedimentos quantitativos como qualitativos de pesquisa.

Entre os agrupamentos que abordaram exclusivamente estratégia em organizações, dois deles contaram com o envolvimento de pesquisadores da UFPR. Um deles foi predominantemente formado por autores dessa instituição, liderado pelo professor Sérgio Bulgacov. O tema principal desse agrupamento refere-se ao estudo da estratégia como processo e como conteúdo. O segundo agrupamento ligado a essa instituição também é composto por autores da FGV/RJ - Fundação Getúlio Vargas, unidade do Rio de Janeiro, em que o tema principal concerne à relação entre estratégia e ambiente organizacional. Mesmo havendo pesquisadores da UFPR nesses dois agrupamentos, trabalhando sob temas similares, não se observou cooperação entre eles. Ainda no Estado do Paraná constatamos a existência de um cluster formado por professores da Pontifícia Universidade Católica do Paraná (PUC-PR), com tema ligado ao posicionamento estratégico em relação com tecnologia. Outro agrupamento que se dedicou quase exclusivamente à estratégia é formado por pesquisadores da Universidade Federal de Santa Catarina (UFSC). O principal autor desse grupo é Cristiano J. C. Cunha, que trabalhou temas concernentes ao estudo de capacidades organizacionais e adaptação estratégica, basicamente sob enfoque qualitativo.

Dois agrupamentos do triênio anterior continuaram atuantes nesse segundo período. O primeiro deles, composto essencialmente por pesquisadores da PUCRio, liderados pelo Professor Jorge Ferreira da Silva, manteve o foco exclusivamente em estratégia, dando continuidade analítica aos temas estratégias competitivas e tipologias estratégicas. O segundo, com foco na confluência entre organizações e estratégia, tem como principal autor o Professor Clóvis L. Machadoda-Silva e colaboradores bastante atuantes como Valéria Silva da Fonseca. Esse grupo, vinculado predominantemente à UFPR, mantém basicamente os mesmos temas de interesse em pesquisa, com maior aprofundamento analítico em termos do uso da perspectiva institucional de análise. $\mathrm{O}$ autor principal do grupo, em especial, defende fortemente a necessidade de articulação e conversação entre os campos de estudos organizacionais e da estratégia em organizações.

O outro agrupamento que trabalha paralelamente as áreas de estudos organizacionais e de estratégia em organizações é formado por pesquisadores da Escola de Administração de Empresas de São Paulo da Fundação Getúlio Vargas 
(EAESP/FGV). Os autores que se destacam são Flávio Carvalho de Vasconcelos, com foco mais específico na confluência da estratégia e organizações, e Maria José Tonelli, Miguel Pinto Caldas e Thomaz Wood Jr. com temas concernentes à gestão estratégica de organizações e pós-modernismo.

Em síntese, observamos, no triênio 2000-2002, crescimento acentuado no número de agrupamentos que privilegiaram temas diferentes. No entanto, a heterogeneidade de temas caracterizou-se como fenômeno entre agrupamentos, uma vez que constatamos grande homogeneidade temática no interior dos agrupamentos, com raras exceções. Os casos isolados de menor nível de homogeneidade intra-agrupamentos ocorreram naqueles em que os grupos encontravam-se conectados de maneira residual, com poucos participantes atuando em vários grupos. Nesses casos, se, ao invés do cluster, consideramos o grupo como unidade de análise, verificamos alto grau de homogeneidade temática.

Muitos grupos que se formaram no triênio anterior persistiram no triênio 20002002. A maioria manteve os temas de preferência; alguns se fragmentaram. Dos três agrupamentos identificados, dois deles também se mantiveram. Apesar do substancial crescimento quantitativo que observamos no campo científico da estratégia em organizações no país, é evidente que a persistência de temas reforça o papel da rede de relações como elemento que proporciona consistência à reprodução das estruturas institucionais, aqui entendidas como práticas sociais duradouras (Giddens, 1989; Machado-da-Silva et al., 2006).

Já no último triênio (2003-2005) constam 1.445 autores, quase o dobro do triênio imediatamente anterior. Novamente, o crescimento proporcional do número de grupos com sete ou mais autores foi bem superior ao crescimento da rede. Identificamos 66 grupos, integrados por 572 pesquisadores (40\% do total da rede), contra 28 do triênio anterior, o que demonstra que a participação dos autores em grupos cada vez maiores constitui tendência merecedora de atenção. A média do tamanho dos grupos também cresceu, apresentando 8,67 autores por grupo. Verificamos, ainda, aumento no quantitativo máximo de integrantes dos grupos: um grupo com 16 autores e dois grupos com 14, em consonância, pois, com a continuada tendência de crescimento no tamanho dos grupos dos triênios anteriores. Observamos grupos com alto grau de similaridade, o que mantém a linha de continuado aumento no nível de permeabilidade e abertura dos grupos. No entanto, alguns poucos grupos revelaram diferenças significativas. A partir da análise de agrupamentos, identificamos 35 clusters nas áreas de estudos organizacionais e estratégia em organizações. Vinte e três deles, que integram o campo científico da estratégia em organizações, podem ser visualizados no Quadro 3. Apesar do grande incremento no número de grupos, muitos deles se sobrepõem em boa medida, o que possibilitou a redução do montante possível de agrupamentos, 
facilitando a análise de um triênio com número tão elevado de autores. Verificamos, também, aumento no quantitativo de clusters com mais de três grupos, o que pode caracterizar a possível emergência de conglomerados de pesquisadores no campo da estratégia em organizações no Brasil.

\section{Quadro 3: Agrupamentos e Principais Temas no Triênio 2003-2005}

\begin{tabular}{|c|c|c|c|}
\hline \begin{tabular}{|l|} 
Instituições \\
Principais
\end{tabular} & Áreas & $\begin{array}{l}\text { Eixo Temático } \\
\text { Predominante }\end{array}$ & nais Abord \\
\hline $\begin{array}{l}\text { UFPR, FGV- } \\
\text { SP }\end{array}$ & $\begin{array}{l}\text { ORG } \\
\text { e ESO }\end{array}$ & TEO e ESO & $\begin{array}{l}\text { Teoria Institucional e Estratégia; Identidade Organizacional; } \\
\text { Cognição e Estratégia; Estratégia e Mudança; Estratégia e } \\
\text { Desempenho; Redes Organizacionais. }\end{array}$ \\
\hline FGV-SP & $\begin{array}{l}\text { ORG } \\
\text { e ESO }\end{array}$ & TEO e ESO & $\begin{array}{l}\text { Vantagem Competitiva e Performance; Redes; Subjetividade e } \\
\text { Gestão de Pessoas; Teoria Institucional. }\end{array}$ \\
\hline $\begin{array}{l}\text { UEM, } \\
\text { UNICENP, } \\
\text { PUC-PR }\end{array}$ & $\begin{array}{l}\text { ESO e } \\
\text { ORG }\end{array}$ & $\begin{array}{l}\text { ESO, EMP e } \\
\text { TEO }\end{array}$ & $\begin{array}{l}\text { Estratégia e Visão Baseada em Recursos; Cultura Organizacional; } \\
\text { Teoria Institucional; Empreendedorismo. }\end{array}$ \\
\hline UECE & $\mathrm{SO}$ & ESO e EMP & dedorismo; Empresas Familiares e Alianças Estr \\
\hline Mackenzie & ESO & ESO & va; Inovação. \\
\hline UFPE & ESO & EMP & $\begin{array}{l}\text { Competências e Comportamento do Empreendedor; Orientação para } \\
\text { o Mercado. }\end{array}$ \\
\hline \begin{tabular}{|l} 
UFMG, \\
UFLA
\end{tabular} & $\begin{array}{ll}\mathrm{G} \\
\text { SO }\end{array}$ & $\begin{array}{lll}\text { TEO, } & \text { COR } & \mathrm{e} \\
\text { ESO } & \end{array}$ & Alianças Estratégicas; Construcionismo e Interpretativismo; \\
\hline UNIFOR & 50 & ESO & Estratégica; Gestão do Conhecimento. \\
\hline Mackenzie & $\begin{array}{l}\text { ORG } \\
\text { e ESO }\end{array}$ & COR e EMP & $\begin{array}{l}\text { Diversidade Cultural; Liderança e Empreendedorismo; } \\
\text { Aprendizagem e Competências. }\end{array}$ \\
\hline UFMG & & ESO e EMP & a; Empreendedorismo. \\
\hline UNIFOR & $\mathrm{O}$ & EMP & Empreendedorismo e Comportamento Empreendedor. \\
\hline $\begin{array}{l}\text { USP e PUC- } \\
\text { SP }\end{array}$ & ESO & ESO e GIN & $\begin{array}{l}\text { Estratégia e Desempenho Financeiro; } \text { Estratégias } \\
\text { Internacionalização. }\end{array}$ \\
\hline UEM & $\begin{array}{l}\text { ORG } \\
\text { e ESO }\end{array}$ & TEO e EMP & edorismo. \\
\hline \begin{tabular}{|l|l} 
UNIVALI \\
UNISUL
\end{tabular} & ESO & ESO e GIN & $\begin{array}{l}\text { Mudança e Adaptação Estratégica; Internacionalização de } \\
\text { Empresas. }\end{array}$ \\
\hline UNISINOS & $\mathrm{O}$ & GIN & ações. \\
\hline USP & $\mathrm{O}$ & ESO & Estratégicas. \\
\hline PUC-MG & ESO & ESO & Responsabili \\
\hline $\begin{array}{l}\text { UFRJ e PUC- } \\
\text { Rio }\end{array}$ & ESO & ESO e GIN & $\begin{array}{l}\text { Estratégia Competitiva; Tipologias Estratégicas; Desempenho; } \\
\text { Desempenho das Exportações; Internacionalização. }\end{array}$ \\
\hline $\begin{array}{l}\text { UFRGS, } \\
\text { UFSM }\end{array}$ & ESO & ESO & Redes Interorganizacionais; Estratégia em Organizações. \\
\hline Mackenzie & ESO & ESO & $\begin{array}{l}\text { Visão Baseada em Recursos; Valor de Ativos; Rendas Econômicas; } \\
\text { Desempenho. }\end{array}$ \\
\hline PUC-PR & ESO & ESO & ia Competitiva. \\
\hline PUC-Rio & ESO & ESO & e Redes Estratégicas; Relacionamentos Estratégicos. \\
\hline UFRGS & ESO & $\begin{array}{lll}\text { ESO, EMP e } & \\
\text { GIN } & & \\
\end{array}$ & $\begin{array}{l}\text { Estratégia de Empresas; Redes de Pequenas Empresas; } \\
\text { Conhecimento e Inovação em Redes; Empresas Multinacionais. }\end{array}$ \\
\hline
\end{tabular}

Fonte: Dados primários da pesquisa.

Em comparação com os triênios anteriores, dos 23 agrupamentos relacionados 17 deles restringiram-se exclusivamente ao campo da estratégia em organizações. Os outros seis clusters circunscreveram sua atuação na confluência do campo da estratégia com a área de estudos organizacionais. Portanto, mesmo diante do 
grande crescimento quantitativo do campo científico em exame, praticamente se manteve a proporção de agrupamentos dedicados exclusivamente à área de estratégia relativamente aos agrupamentos focados na junção entre estratégia e estudos organizacionais.

Dos agrupamentos interessados tão somente no campo exclusivo da estratégia, oito deles situam-se especificamente no eixo da área temática de estratégia em organizações, em consonância com a classificação da ANPAD em 2005. Tal classificação não deixa de ser problemática, uma vez que, ao segmentar o campo em três subconjuntos, atribui a um deles designação idêntica ao do próprio conjunto como um todo.

Do total de 23 agrupamentos, dois deles estão ligados à Universidade Presbiteriana Mackenzie. Um deles liderado por Moisés Zilber, que estudou predominantemente os temas estratégia e vantagem competitiva, e inovação em setores industriais. Já o segundo agrupamento, teve como pesquisadores principais Eduardo Ulrich Pace, Herbert Kimura, Leonardo Fernando Cruz Basso e Wilson Toshiro Nakamura. Os autores utilizaram, sistematicamente, modelos de simulação em seus trabalhos, focando temas tais como: visão baseada em recursos, valor de ativos, rendas econômicas e desempenho. Apesar de pertencerem à mesma instituição de ensino superior, a abordagem metodológica atua como elemento diferenciador entre esses dois agrupamentos.

Além dos dois anteriores, três outros clusters trabalharam praticamente com o mesmo tema, isto é, estratégia e vantagem competitiva. Eles estão vinculados a três instituições distintas: Universidade de São Paulo (USP), Universidade de Fortaleza (UNIFOR), e PUC-PR. Apesar do tema focal circular em torno de estratégia competitiva, ao que parece a distância entre as instituições de vínculo foi primordial para a não ocorrência de conexão entre tais agrupamentos.

Dois outros agrupamentos dedicaram-se ao estudo de alianças estratégicas e relacionamentos interorganizacionais. Um deles ligado à PUC-Rio; outro vinculado a duas instituições do sul do país: Universidade Federal do Rio Grande do Sul (UFRGS) e Universidade Federal de Santa Maria (UFSM). O cluster da PUCRio foi liderado por T. Diana L. V. A. de Macedo-Soares e o da UFRGS e UFSM por Breno Augusto Diniz Pereira e Eugênio Ávila Pedrozo. Sob perspectiva um tanto quanto diferenciada e apesar de apresentar pequena produção, o agrupamento conduzido por Roberto Patrus Mundin Pena, vinculado à Pontifícia Universidade Católica de Minas Gerais (PUC-MG) e à Fundação Dom Cabral (FDC), endereçou o tema da ética e da responsabilidade social no campo da estratégia.

Identificamos mais seis clusters com foco no eixo temático de estratégia em organizações, mas já dividindo espaço com os temas gestão internacional e 
empreendedorismo. Entre os que trabalharam estratégia em relação com o tema de empreendedorismo, observamos um agrupamento na Universidade Estadual do Ceará (UECE), liderado por Ana Freitas; outro na Universidade Federal de Minas Gerais (UFMG) sob a coordenação de Carlos Alberto Gonçalves; e um terceiro na UFRGS, liderado por Dirk Michael Boehe, Karen Menger da Silva, Lilia Maria Vargas e Paulo Antônio Zawislak. O interesse principal entre tais agrupamentos relaciona-se tanto ao tema de empreendedorismo como à estratégia competitiva em si. O agrupamento da UECE também revelou interesse no estudo de empresas familiares e alianças estratégicas, diferenciando-se, assim, dos outros dois. Já o agrupamento ligado a UFRGS registra amplo interesse em redes de pequenas empresas, bem como no estudo de empresas internacionais.

Ainda no que concerne aos agrupamentos que se interessaram pelo tema de gestão internacional, dentre aqueles mais centrados na área de estratégia, o quarto deles, vinculado à USP e à PUC-SP, tem como interesse principal questões de desempenho financeiro e de estratégias de internacionalização. Felipe Borini (USP) e Eduardo Proença (PUC-SP) são os principais pesquisadores desse agrupamento. Um quinto agrupamento que estuda o processo de internacionalização em relação com a estratégia localiza-se em Santa Catarina, mais especificamente na UNIVALI e UNISUL, com foco em temas tradicionais de internacionalização e mudanças estratégicas.

O sexto agrupamento atuante na confluência de estratégia em organizações e gestão internacional tem como pesquisadores principais Angela da Rocha e Carlos Alberto Hemais, ambos da Universidade Federal do Rio de Janeiro (UFRJ), e Jorge Ferreira da Silva da PUC-Rio. Este último autor, que se mantém desde o primeiro triênio como um dos mais reconhecidos estudiosos no campo da estratégia, aproximou-se dos outros dois pesquisadores mais recentemente, o que implicou que seu grupo, que trabalha fortemente a perspectiva clássica da estratégia, abrisse o leque de temas sobre internacionalização, porém sempre por meio de métodos estatísticos de análise. O único agrupamento que trabalhou predominantemente com o tema de internacionalização está localizado na UNISINOS, sendo liderado por Marcelo André Machado, com foco em exportações de empresas brasileiras.

No que se refere ao eixo temático sobre empreendedorismo, encontramos dois agrupamentos. Um deles localizado na Universidade Federal de Pernambuco (UFPE), tendo como principais pesquisadores Fernando Paiva Júnior e Sérgio Benício de Mello. O outro é liderado por Ana Sílvia Rocha Ipiranga da Universidade de Fortaleza (UNIFOR). Ambos os agrupamentos têm como tema o comportamento empreendedor. No entanto, o primeiro deles procura avaliar as competências do empreendedor e, ainda, questões vinculadas à orientação para o mercado de pequenas empresas. 
Entre os agrupamentos que adotaram como foco exclusivamente a área de estratégia, verificamos a predominância de perspectivas mais tradicionais ligadas à vantagens e estratégias competitivas e aos recursos da firma. Valoriza-se uma abordagem mais voluntarista da estratégia, levando em conta fortemente a influência da racionalidade nas decisões estratégicas.

Já nos agrupamentos que abordam a estratégia sob uma perspectiva organizacional, verificamos algumas diferenças de foco do triênio imediatamente anterior para o atual. Dos seis agrupamentos que se interessaram tanto pela área de Estratégia como pela área de Organizações, dois deles se concentraram na confluência entre elas. Um deles é formado basicamente por pesquisadores da UFPR e da FGV-SP, com a participação adicional de alguns pesquisadores da PUC/PR, do Centro Universitário Positivo (UnicenP) e da Universidade Estadual de Maringá (UEM). O outro é integrado exclusivamente por pesquisadores da FGV-SP.

No primeiro agrupamento, os autores mais destacados são Clóvis L. Machadoda-Silva, que se mantém consistentemente no mesmo eixo temático desde o primeiro triênio, Flávio Carvalho de Vasconcelos, Valéria Silva da Fonseca, João Marcelo Crubellate e Fernando Gimenez. O foco principal desse agrupamento é a teoria institucional. Procuram enfatizar a relação entre estrutura e ação, considerando que a estratégia é mais bem entendida sob uma ótica que não separa os estudos organizacionais da estratégia em organizações. Redes organizacionais com base analítica da teoria institucional também tem sido objeto de investigação por pesquisadores desse agrupamento. Vários autores desse grupo, em especial Clóvis L. Machado-da-Silva e João Marcelo Crubellate, enfatizam, mais recentemente, a noção de estratégia como prática. Já Flávio Carvalho de Vasconcelos e alguns colaboradores exploram questões ligadas ao desempenho de empresas.

O segundo agrupamento, formado especialmente por pesquisadores da FGVSP, tem como principais colaboradores Carlos Osmar Bertero, Flávio Carvalho de Vasconcelos, Isabella Freitas Gouveia de Vasconcelos, Luiz Artur Ledur Brito e André Ofenhejm Mascarenhas. Dois autores externos também participam: Eliane Pereira Zamith Brito (Mackenzie) e João Marcelo Crubellate (UEM). Na área de Estratégia, os temas mais abordados são: vantagem competitiva, desempenho e redes organizacionais. Como esses dois agrupamentos apresentaram grande quantitativo de autores e grande número de grupos, observamos certa heterogeneidade de temas entre ambos. Todavia, no que se refere aos outros agrupamentos, verificamos que esses mesmos autores, em alguns casos, encontram-se agrupados sob um foco mais restrito.

Um terceiro agrupamento, que tratou estratégia e teoria das organizações de maneira bem próxima, mas com foco mais restrito, é composto por pesquisadores 
da UEM, do UnicenP e da PUC-PR. Paulo Grave, João Marcelo Crubellate e Fernando Gimenez são os seus principais autores. No entanto, Fernando Gimenez apresenta interesse adicional ligado ao eixo temático de empreendedorismo, que não é efetivamente compartilhado pelos outros dois autores. Ainda na UEM, encontramos um quarto agrupamento, de menor porte, conduzido por Hilka Vier Machado, que adota como foco de suas pesquisas temas como empreendedorismo e identidade organizacional.

O quinto agrupamento, que trabalhou tanto teoria das organizações como estratégia em organizações, procurou estudar o tema comportamento organizacional. Constitui um dos maiores agrupamentos do período, formado por seis grupos, com autores oriundos da Universidade Federal de Minas Gerais (UFMG) e da Universidade Federal de Lavras (UFLA). Entre os autores principais desse grupo estão Marlene Catarina de Oliveira Lopes Melo, Mônica Carvalho Alves Cappelle, Mozar José de Brito e Elcemir Paço-Cunha. Os temas variam desde redes e alianças estratégicas até construcionismo, interpretativismo e gênero.

Por fim, o sexto e último agrupamento, que se dedicou às duas áreas simultaneamente, tem como foco dois temas que não haviam sido trabalhados em conjunto até então: comportamento organizacional e empreendedorismo. Esses estudos foram principalmente levados a efeito por Maurício Henrique Benedetti e Vânia Maria Jorge Nassif, ambos da Universidade Presbiteriana Mackenzie. Eles procuram pesquisar, sob a ótica comportamental, questões ligadas à diversidade cultural, aprendizagem e competências. Ainda sob a perspectiva comportamentalista, os autores estudaram a liderança e o empreendedor, aproveitando as abordagens utilizadas tradicionalmente por aqueles que consideram o comportamento do empreendedor como chave para o sucesso.

Em linhas gerais, verificamos aumento significativo no montante de grupos e de agrupamentos. Esse aumento é bem superior ao crescimento da rede, refletindo o maior número de laços que os autores vêm desenvolvendo em cada triênio. A investigação dos agrupamentos contribuiu para compreendermos como a delimitação estrutural das relações entre autores condiciona a construção do conhecimento científico. Autores imersos em agrupamentos são mais suscetíveis de influenciarem e de serem influenciados em sua visão de ciência pelos padrões ali adotados (Leydesdorff, 2007; Moody, 2004).

Os temas constantes dos agrupamentos que consideram a junção ou a articulação da área de estratégia com a área de organizações refletem a influência de perspectivas de análise mais diversas. O exame do fenômeno da estratégia com base em perspectivas oriundas do campo dos estudos organizacionais oferece alternativas interessantes. Por exemplo, alguns pesquisadores interessados em teoria institucional procuram avaliar a estratégia sob esse enfoque, enfatizando o 
papel da estrutura, da agência e da interpretação intersubjetiva dos atores sociais. Já aqueles pesquisadores que se orientam por uma visão subjetiva da realidade enfocam a estratégia mais como resultante da interpretação subjetiva, supervalorizando o individualismo metodológico. Em face dessas constatações, observamos que o conjunto de crenças e valores sobre o qual esses autores se apóiam em sua área de origem claramente afetam a leitura que fazem da realidade, uma vez que condicionam sua própria visão de ciência e do fazer científico.

\section{CONCLUSÃo}

A partir de uma análise longitudinal, procuramos, no presente artigo, aproximar o entendimento da dinâmica das redes de relacionamentos com o desenvolvimento do campo científico da estratégia em organizações. Para tanto, utilizamos a produção científica dos autores na área de estratégia, a fim de compreender como é exercida a capacidade de agência desses autores à luz do exame de sua recursividade com a estrutura de relações dos sistemas sociais. Com base no pressuposto de que há ligação entre a microdinâmica do comportamento dos autores em nível local com as propriedades globais da rede de relacionamento, direcionamos o foco de nossa análise para a persistência e a mudança de temas na estruturação do campo científico da estratégia, no período compreendido entre 1997 e 2005.

A análise dos dados evidenciou que o campo científico da estratégia em organizações no âmbito da academia brasileira de administração não é amplamente conectado. Como tal, apresenta grande número de autores e grupos que não cooperam direta ou indiretamente entre si. Em face desses achados, podemos considerar o campo como altamente fragmentado no tocante ao nível de imersão estrutural dos pesquisadores, do qual decorre a grande heterogeneidade de temas que observamos no decorrer dos nove anos de análise.

Apesar dessa constatação e com base no que nos ensinaram autores como DiMaggio e Powell (1983) e Scott (1994, 2001), podemos afirmar que o campo científico da estratégia em organizações compreende uma área reconhecida da vida institucional, na qual os autores compartilham sistemas de significados comuns, possibilitando a ocorrência de isomorfismo entre os participantes do campo. Nesse sentido, entendemos que os autores de textos acadêmicos sobre estratégia compartilham certas crenças sobre a noção de ciência e do fazer científico, mesmo em face de um grande número de atores e grupos isolados, o que implica na possibilidade de conversação entre perspectivas analíticas do campo, levando os autores à monitoração reflexiva no sistema social. 
No que concerne às medidas de coesão estrutural com base na identificação dos grupos e dos agrupamentos na rede de relacionamento, verificamos a ocorrência de significativo aumento no número de grupos no período em exame. Tal incremento demonstrou ser bem superior ao crescimento quantitativo da rede, refletindo o maior número de laços que os autores vêm apresentando de um triênio para outro no decorrer do período como um todo. Mediante análise de conteúdo dos agrupamentos identificados, constatamos a existência de homogeneidade de temas dentro de cada agrupamento, o que reforça a afirmativa de que o nível de coesão entre os autores influencia o que eles consideram relevante como conteúdo a ser estudado e desenvolvido (Hanneman \& Riddle, 2005; Moody, 2004). Assim, o nível de coesão estrutural entre autores constitui um facilitador de práticas isomórficas de pesquisa, o que possibilita a construção de regras e de normas científicas (Scott, 2001). Essas regras e normas estão vinculadas não apenas aos grupos de pesquisadores, mas também a organizações existentes em diferentes esferas e níveis da atividade científica, tais como programas de pósgraduação stricto sensu, associações científicas, órgãos de fomento à pesquisa e produção intelectual, instituições universitárias, e assim por diante.

Se no interior dos grupos observamos alto grau de homogeneidade no que concerne aos temas de orientação para pesquisa, ao considerarmos o conjunto deles, na amplitude do campo da estratégia em organizações, constatamos a predominância da heterogeneidade de temas. Todavia, mesmo em face da significativa heterogeneidade no campo, os autores tendem a trabalhar nos temas adotados somente no interior dos próprios grupos. Tal evidência significa que os grupos estão frouxamente articulados.

Boa parte dos grupos surgidos nos triênios anteriores persistiu no período como um todo: alguns mantiveram os mesmos temas; outros se fragmentaram por novos temas. Constatamos, assim, que apesar do significativo crescimento do campo da estratégia em organizações como um todo, a maioria dos temas de triênios anteriores permaneceu no período, o que demonstra que a rede de relações provê certa consistência à (re)produção das estruturas institucionais, aqui entendidas como práticas sociais duradouras, no sentido de durabilidade dinâmica em oposição a concepções deterministas estáticas (Giddens, 1989; Machado-da-Silva et al., 2006).

Já no que se refere especificamente aos agrupamentos, concluímos que os temas adotados no triênio anterior influenciaram as temas de pesquisas em momentos posteriores, ocasionando baixa diversidade de temas no interior dos agrupamentos. Tal circunstância está por merecer análise mais aprofundada, uma vez que não constitui objetivo do presente artigo, no sentido de verificar se ocorreu algum nível de contribuição para a acumulação de conhecimento no campo 
científico da estratégia em organizações. Nos poucos casos de agrupamentos com maior heterogeneidade de temas, observamos que resultou principalmente do tamanho dos agrupamentos.

Ainda sobre a análise dos agrupamentos e seus respectivos eixos temáticos, algumas considerações se fazem necessárias. A primeira delas é relativa à grande heterogeneidade de temas no campo como um todo. Fuchs (1993) levanta duas importantes questões sobre a natureza do trabalho dos pesquisadores: a dependência mútua e a incerteza das tarefas. $\mathrm{O}$ autor considera que, em campos científicos com várias fontes de recursos, ganha espaço a tendência de redução na dependência mútua entre autores, possibilitando a formação de 'adhocracias fragmentadas'. Ainda que se verifiquem relações entre os diferentes grupos no campo científico eles tendem a estar frouxamente conectados, o que permite que cada um deles persista e desenvolva sistemas de significado comuns no interior do grupo, que não são necessariamente compartilhados no campo como um todo.

No caso da incerteza das tarefas, o autor considera que, diferentemente das ciências físicas, os resultados das pesquisas nas ciências sociais são difíceis de serem interpretados, em decorrência de controvérsias e ambigüidades. Em face da baixa dependência entre os grupos e da incerteza das tarefas, Fuchs (1993) afirma que as ciências sociais tendem a ser mais fragmentadas do que campos científicos nos quais as tarefas são mais rotinizadas em poucos centros de pesquisa. Apesar da grande heterogeneidade de temas, a formação de grupos no campo de pesquisa em estratégia claramente possibilitou maior homogeneidade de práticas de pesquisa, implicando em algum grau de conversação entre pesquisadores.

A imersão social em grupos pode ser avaliada tanto nos aspectos estruturais como nos simbólicos, conforme destacam Moody e White (2003). Comparando as abordagens intra e inter grupos, verificamos que a constituição de grupos e de clusters serviu como elemento de homogeneização de temas de investigação, que foram compartilhados pelos autores pertencentes a cada grupo ao tempo que, entre grupos diferentes, observamos a tendência de diferenciação entre temas. Em alguns casos de perspectivas mais tradicionais os temas coincidiram na amplitude do campo, mas decorrem do caráter clássico dessas abordagens.

As preferências teóricas na escolha de temas constituem evidências de sistemas de significado compartilhados pelos autores, indicando o que eles entendem como ciência no campo. Assim, observamos o compartilhamento de crenças e pressupostos que interferiram na construção social do conhecimento científico na área de estratégia. Em face das evidências estruturais (coesão estrutural) e simbólicas (autores que adotam temas similares em seus grupos) podemos, em síntese, afirmar que houve convergência entre a formação de grupos estruturados e os temas desenvolvidos em cada grupo, e distinção quando comparados com os 
grupos de fora. Dessa forma, é plausível afirmar que a estrutura de relações condicionou, para o bem e para o mal, os temas desenvolvidos no campo de conhecimento da estratégia em organizações.

\section{Artigo recebido em 26.02.2007. Aprovado em 04.07.2007.}

\section{NotA}

${ }^{1}$ Este artigo resulta de uma agenda de estudos em processo no âmbito do grupo de pesquisa cadastrado no CNPq sob a designação "Estudos Organizacionais e Estratégia"; além disso, integra um programa de pesquisas sobre a "Institucionalização da Pós-Graduação em Administração no Brasil”, financiado pelo PROCAD/CAPES.

\section{RefERÊNCIAS BibliográficAs}

Batagelj, V., \&

Mrvar, A. (2005).

PAJEK - Program for analysis and visualization of large networks. Ljubljana, Slovenia: University of Ljubljana.

Borgatti, S. P.,

Everett, M. G., \&

Freeman, L. C. (2002).

UCINET for Windows: Software for Social Network Analysis. Boston: Harvard Analytic Technologies.

De Nooy, W. (2003).

Fields and networks: correspondence analysis and social network analysis in the framework of field theory. Poetics, 31(5-6), 305-327.

Déry, R., \&

Toulouse, J. (1996).

Social structuration of field of entrepreneurship: a case study. Canadian Journal of Administrative Sciences, 13(4), 285-305.
DiMaggio, P. J., \&

Powell, W. W. (1983).

The iron cage revisited: institutional isomorphism and collective rationality in organizational fields. American Sociological Review, 48(2), 147-60.

Freeman, L. C. (1992).

Social networks and the structure experiment. In L. C. Freeman, D. R. White, \& K. A. Romney (Eds.). Research Methods in Social Network Analysis (pp. 11-40). New Brunswick, NJ: Transaction Publishers.

Friedkin, N. E. (1998).

A structural theory of social influence. Cambridge: Cambridge Univesity Press.

Fuchs, S. A. (1993).

Sociological theory of scientific change. Social Forces, 71(4), 933-953.

Giddens, A. (1978).

Novas regras do método sociológico. Rio de Janeiro: Zahar. 
Giddens, A. (1989).

A constituição da sociedade. São Paulo: Martins Fontes.

Granovetter, M. S. (1985).

Economic action and social structure: the problem of embeddedness. American Journal of Sociology, 91(3), 481-510.

Hanneman, R. A., \&

Riddle, M. (2005).

Introduction to social network methods. Riverside: University of Califórnia.

Kuhn, T. S. (1978).

A estrutura das revoluções científicas. São Paulo: Perspectiva.

Leydesdorff, L. (2007).

Scientific communication and cognitive codification: social systems theory and the sociology of scientific knowledge. European Journal of Social Theory, 10(3), 1-22.

Liberman, S., \&

Wolf, K. B. (1997).

The flow of knowledge: scientific contacts in formal meetings. Social Networks, 19(3), 271-283.

Machado-da-Silva, C. L.,

Fonseca, V. S., \&

Crubellate, J. M. (2005).

Unlocking the institutionalization process: insights for an institutionalizing approach. Brazilian Administration Review, 2(1), 1-20.

Machado-da-Silva, C. L.,

Guarido Filho, E. R., \&

Rossoni, L. (2006).

Organizational fields and the structuration perspective: analytical possibilities. Brazilian Administration Review, 3(2), 32-56.

Moody, J. (2004).

The structure of a social science collaboration network: disciplinary cohesion from 1963 to 1999. American Sociological Review, 69(2), 213-238.

Moody, J., \&

White, D. R. (2003).

Structural cohesion and embeddedness: a hierarchical concept of social groups. American Sociological Review, 68(1), 103-127.

Popper, K. (1972).

Conjecturas e refutações. Brasília: UNB.

Popper, K. (1989).

A lógica da pesquisa científica (4a ed.). São Paulo: Cultrix.

Powell, W. W.,

White, D. R.,

Koput, K. W., \&

Owen-Smith, J. (2005).

Network dynamics and field evolution: the growth of interorganizational collaboration in the life sciences. American Journal of Sociology, 110(4), 1132-1205.

Scott, W. R. (1994).

Conceptualizing organizational fields: linking organizations and societal systems. In H. Derlien, U. Gerhardt, \& F. W. Scharpf (Eds.). Systems Rationality and Partial Interests (pp. 203-221). Baden: Nomos.

Scott, W. R. (2001). Institutions and organizations $(2 \mathrm{a}$ ed.). Thousand Oaks: Sage. 
Wagner, C. S., \&

Leydesdorff, L. (2005).

Network structure, self-organization, and the growth of international collaboration in science. Research Policy, 34(10), 1608-1618.

Wasserman, S., \&

Faust, K. (1994).

Social network analysis: methods and applications. Cambridge: Cambridge University Press.
White, D. R.,

Owen-Smith, J.,

Moody, J., \&

Powell, W. W. (2004).

Networks, fields and organizations: micro-dynamics, scale and cohesive embeddings. Computational \& Mathematical Organization Theory, 10(1), 95-117. 